\title{
The Manhattan and Lorentz Mirror Models: A Result on the Cylinder with Low Density of Mirrors
}

\author{
Kieran Ryan ${ }^{1}$ (D) \\ Received: 9 November 2020 / Accepted: 3 September 2021 / Published online: 13 October 2021 \\ (c) The Author(s) 2021
}

\begin{abstract}
We study the Manhattan and Lorentz mirror models on an infinite cylinder of finite even width $n$, with the mirror probability $p$ satisfying $p<C n^{-1}, C$ a constant. We show that the maximum height along the cylinder reached by a walker is order $p^{-2}$. We observe an algebraic structure, which helps organise our argument. The models on the cylinder can be thought of as Markov chains on the Brauer (in the Mirror case) or Walled Brauer (in the Manhattan case) algebra, with the transfer matrix given by multiplication by an element of the algebra.
\end{abstract}

Keywords Probability $\cdot$ Statistical mechanics $\cdot$ Random walks $\cdot$ Manhattan model $\cdot$ Lorentz mirror Model $\cdot$ Brauer algebra

\section{Introduction}

The Manhattan and Lorentz mirror models [2,10], are two very similar models, each describing a random walk on the $\mathbb{Z}^{2}$ lattice. Let $0 \leq p \leq 1$. The walker is a particle of light which bounces off mirrors placed at each vertex at $45^{\circ}$, independently with probability $p$. For the Lorentz mirror model, the orientation of the mirror (NW or NE) is chosen independently with probability $\frac{1}{2}$. For the Manhattan model, the lattice is a priori given Manhattan directions (see Fig. 1), and the orientation of the mirror is determined by its location (i.e. a NW mirror if the sum of the coordinates of the point is odd, and NE if the sum is even), so that the walker always follows the directions of the lattice. The main questions of interest in both models are whether the paths remain bounded or not, and the nature of the motion of the walker.

We study the models on an infinite cylinder $\mathbb{Z} \times(\mathbb{Z} / n \mathbb{Z})$ of finite even width $n$. We are interested in how the length of the paths vary with $p$. Note that on the cylinder, paths are bounded with probability 1 - indeed, there is a positive probability that a horizontal row is

Communicated by Eric A. Carlen.

This study was supported in part by the European Research Council starting Grant 639305 (SPECTRUM).

$凶$ Kieran Ryan

kieran.ryan@qmul.ac.uk

1 Department of Mathematics, Queen Mary University of London, Mile End Road, London E1 4NS, UK 

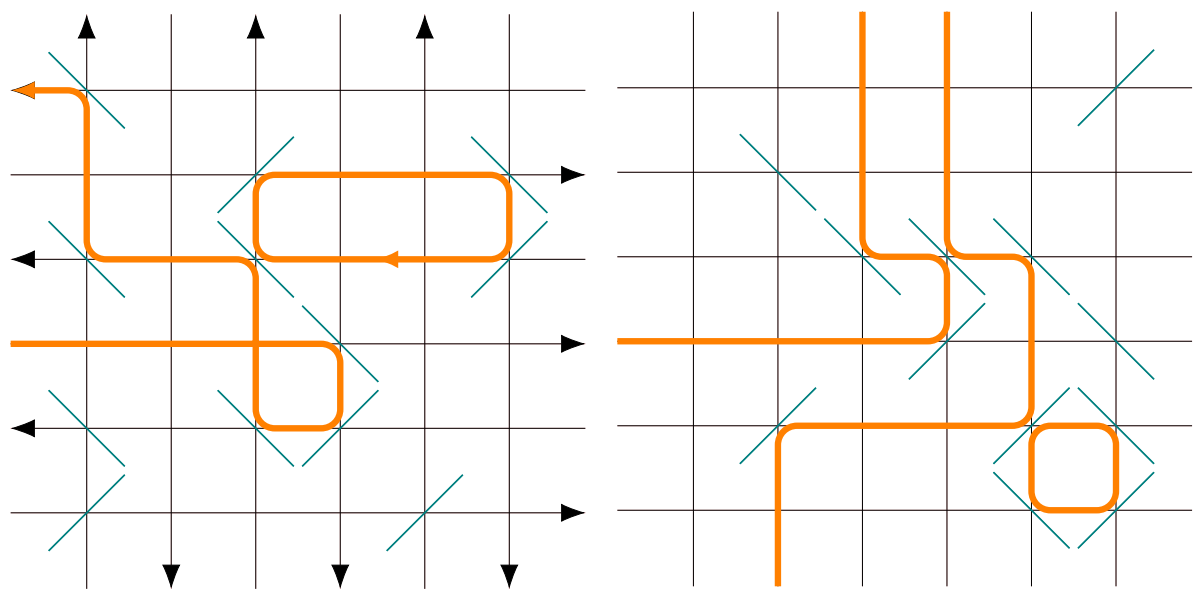

Fig. 1 Examples of the Manhattan model (left) and Mirror model (right), with mirrors in blue, and a few paths highlighted in orange. Note that the orientation of a mirror in the Manhattan case is determined by the Manhattan directions of the lattice (Color figure online)

filled with mirrors such that no path can pass the row; one has to wait an expected $p^{-n}$ rows for this event. It is natural to hope that this bound can be improved. The result of this paper, Theorem 1.1, shows that for both models, when $p \leq C n^{-1}, C$ a constant, the highest row reached by a path on the $n$-cylinder is order $p^{-2}$. We wonder whether this is true for all $p$.

We observe an underlying algebraic structure (valid for any value of $p$ ). The models on the cylinder can be thought of as Markov chains on the Brauer algebra (in the mirror case), or its subalgebra the Walled Brauer algebra (in the Manhattan case). While the result of this paper can be obtained without these algebras, we suggest that the models' association with different algebraic structures reflects their different behaviours. A third model, on the L-lattice (see [1]), with different behaviour from the other two models, is solved using percolation, and can be similarly thought of as a Markov chain on the (extended) Temperley Lieb algebra.

Let us recap the existing results on both models (which are on $\mathbb{Z}^{2}$, unless otherwise specified). The Mirror model was introduced by Ruijgrok and Cohen [16] as a lattice version of the Ehrenfest wind-tree model. Grimmett [9] proved with a straightforward argument that on $\mathbb{Z}^{2}$, if $p=1$, then the path of the walker is bounded with probability 1 . It is conjectured that this is also true for $0<p \leq 1$. This is supported by numerical simulations, for example, in [19]. More recently, Kozma and Sidoravicius [10] showed that, for any $0<p \leq 1$, the probability the walker reaches the boundary of the $n$-box $[-n, n]^{2}$ is at least $\frac{1}{2 n+1}$. To obtain this result, they study the model on an infinite cylinder of finite odd width, where there is deterministically always an infinite path. The Manhattan model cannot be neatly defined on a cylinder of odd width (it cannot remain rotation-invariant), so this method cannot be applied (and indeed, the result is not true in the Manhattan case - see below).

The Mirror model on the cylinder (often under the name the $\mathrm{O}(1)$ loop model) has been studied using the Brauer algebra before, in several papers relating to a conjecture (and variations thereof) by Razumov and Stroganov [7,8,15], which gives the entries of the limiting distribution in terms of combinatorial objects such as alternating sign matrices. A generalised mirror model (the $O(q)$ loop model), where the distribution on configurations is weighted by $q^{\# l o o p s}, q \in \mathbb{C}$, is studied in $[13,14]$; this is the model on the Brauer algebra with parameter 
Fig. 2 The mirrors (in blue) in the Manhattan model as edges in Bernoulli percolation. The green edges form the dual clusters. The two paths shown are restricted to stay within $\frac{1}{\sqrt{2}}$ of one dual cluster (Color figure online)

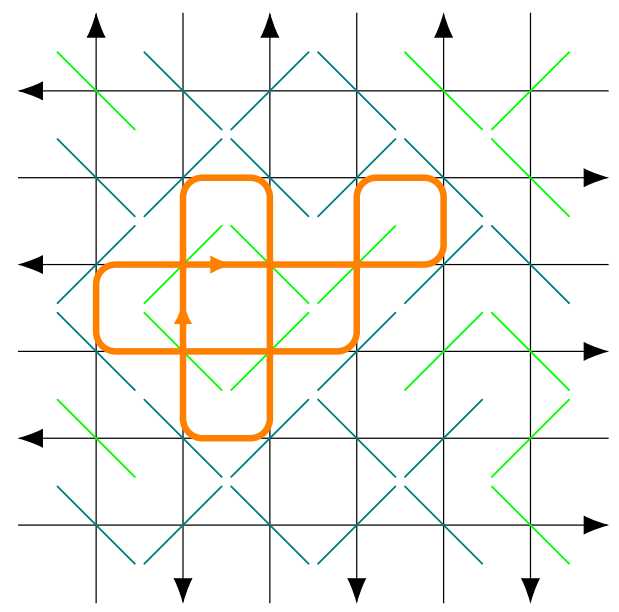

$q, \mathbb{B}_{n, q}$. In these papers, the requirement of a Yang-Baxter equation restricts the permissible values of the parameters - in our specific setup, only $p=\frac{8}{9}$ qualifies (see the end of [14]).

The Manhattan model shares features of quantum disordered systems. The model was introduced by Beamond et al. [1], in close relation to a quantum network model on the Manhattan lattice. The quantum model has random $S p(2)=S U(2)$ matrices on each edge of the lattice, and the classical model arises on averaging over this disorder. In most classical models in two dimensions, localisation (bounded paths) is not observed, whereas in the Manhattan (and Mirror) model, it is expected (see below). It is not clear if the mirror model has a similar explicit relationship with a quantum model. For more detail on the connection to quantum models, see Spencer's review [17].

An argument from [1] for tackling the Manhattan model uses percolation. The placement of the mirrors is exactly a Bernoulli percolation on the edges of $\mathbb{Z}^{2}$, rotated $45^{\circ}$ and scaled. The path of the walker stays within $\frac{1}{\sqrt{2}}$ of its closest dual cluster (see Fig. 2). The dual clusters are finite with probability 1 for $p \geq \frac{1}{2}$, so so are the Manhattan paths.

For $p>\frac{1}{2}$, the probability that two points are in the same dual cluster decays exponentially in the distance, which gives the same for connection by a Manhattan path. This is markedly different from the Mirror model's polynomial decay. For $p<\frac{1}{2}$, this argument is wholly inconclusive, since dual clusters are almost surely infinite. Recently, Li [11] gave exponential decay in connection probabilities for $p>\frac{1}{2}-\epsilon$, for some $\epsilon>0$. Numerical simulations in [2] indicate that paths are finite for $0<p<\frac{1}{2}$, with exponential decay in connection probabilities. Clearly for $p=0$, the paths escape in straight lines to infinity.

On the cylinder, for both models, there is first the crude, simple bound given above. Notably, after this paper was originally posted, $\mathrm{Li}$ [12] showed the following: for both models, on the cylinder of even width $n$, and for fixed $p$, the walker reaches at most $O\left(n^{10}\right)$ rows from its startpoint, with probability exponentially close to 1 . Let us note that the results on the (even) cylinder (including this paper) are the same for the two models, but on $\mathbb{Z}^{2}$ they are different. To analyse the $\mathbb{Z}^{2}$ case via the cylinder, one must look at a cylinder of equal height and width (as in [10]); it is here that the models differ.

Let us now state our result more precisely. Consider the models on the $n$-cylinder $\mathbb{Z} / n \mathbb{Z} \times \mathbb{Z}=\{(i, t): i, t \in \mathbb{Z}, 1 \leq i \leq n\}$, with $n$ even. We label $s_{t}$ the horizontal row $\{(i, t): 1 \leq i \leq n\}$ - the " $t^{t h}$ street". For the Mirror model, let $V_{\frac{n}{2}}^{\text {mir }}$ be the random variable 
given by the smallest $t$ such that $s_{t}$ has no path connecting it to the first street, $s_{1}$. In other words, the highest street a path from $s_{1}$ reaches is exactly $V_{\frac{n}{2}}^{m i r}-1$. Let $V_{\frac{n}{2}}^{\text {mat }}$ be defined identically for the Manhattan model.

Theorem 1.1 Let $*$ represent mat or mir.

(a) Let $\mathrm{p} \leq \mathrm{Cn}^{-1}, \mathrm{C}>0$ a constant. For all $\alpha>0$,

$$
\mathbb{P}\left[V_{\frac{n}{2}}^{*} \geq \alpha p^{-2}\right] \leq 2 A_{*} e^{-\frac{1}{8 e^{C}} \alpha},
$$

where $A_{\text {mir }}=\cosh (\pi)$, and $A_{\text {mat }}=\frac{\sinh (\pi)}{\pi}$.

(b) For any $p \leq \frac{1}{2}$ (not necessarily constrained by $p<C n^{-1}$ ), and for all $\alpha>0$,

$$
\mathbb{P}\left[V_{\frac{n}{2}}^{*} \leq \alpha p^{-2}\right] \leq 2 \alpha .
$$

Let us give an informal overview of the proof of part (a). Our argument uses the streets which have at most two mirrors. As $n \rightarrow \infty$, for all $p \leq C n^{-1}$, and $C$ small, the probability of mirrors is small, and in particular, the probability that each street $s_{t}$ has at most two mirrors is large. We show that the model is not changed too much if we actually condition on each $s_{t}$ having at most two mirrors. This conditioning simplifies the model greatly, in essence removing the cylindrical geometry, making the interactions on each street mean-field (in the sense that if the particle arrives at street $s_{t}$ at the point $(i, t)$ and leaves from $(j, t), j \neq i$, then $j$ is uniformly distributed). This allows us to do explicit computations. For $C$ not small, the theorem still holds, but the bounds are less sharp; one needs to set $\alpha$ exponentially large in $C$ to bring the bound to less than 1. Part (b) is more straightforward; it is proved by coupling $V_{\frac{n}{2}}^{*}$ with a geometric random variable $G$ with parameter $p^{2}$.

In Sect. 2, we give key definitions, including the Brauer and Walled Brauer algebras. In Sect. 3 we study the model assuming at most two mirrors per street, and obtain the results needed to prove Theorem 1.1.

\section{Definitions, and the Brauer Algebra}

Let us define the algebraic structures and notation we will use. The Brauer algebra $\mathbb{B}_{n, 1}$ (see, for example, $[3,4,6,18]$ ) is the (formal) complex span of the set of pairings of $2 n$ vertices. We think of pairings as graphs, which we will call diagrams, with each vertex having degree exactly 1 . We arrange the vertices in two horizontal rows, labelling the upper row (the northern vertices) $1^{+}, 2^{+}, \ldots, n^{+}$, and the lower (southern) $1^{-}, \ldots, n^{-}$. We call an edge connecting two northern vertices (or two southern) a bar. The number of bars in the north and south is always the same, and we refer to either simply as the number of bars in the diagram. We call an edge connecting a northern and southern vertex a NS path.

Multiplication of two diagrams is given by concatenation. If $b, c$ are two diagrams, we align the northern vertices of $b$ with the southern of $c$, and the result is obtained by removing these middle vertices. See Fig. 3 . We let $b c$ denote the product (occasionally $b \cdot c$ for clarity). This defines $\mathbb{B}_{n, 1}$ as an algebra. In general there is a multiplicative parameter $q$, for any $q \in \mathbb{C}$, but for our purposes we only need $q=1$, which gives the multiplication described above. See [3] for more detail.

We call the set of all diagrams $B_{n}$. We call the set of diagrams with exactly $k$ bars $B_{n}\langle k\rangle$, and the set of diagrams with at least $k$ bars $B_{n}^{k}$. Notice that $B_{n}\langle 0\rangle$ is exactly the symmetric 

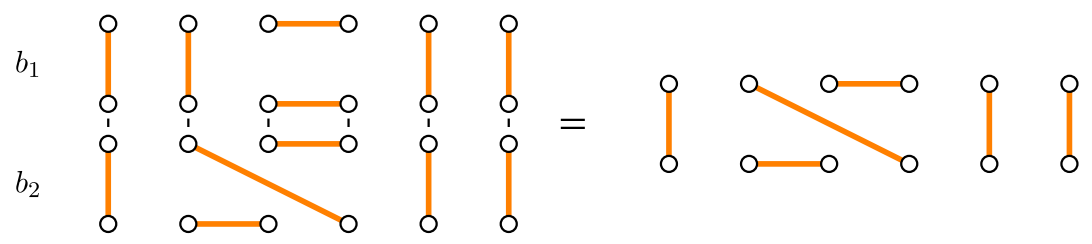

$b_{1} b_{2}$

Fig. 3 Two diagrams $b_{1}$ and $b_{1}$ (left), concatenated to produce their product (right)

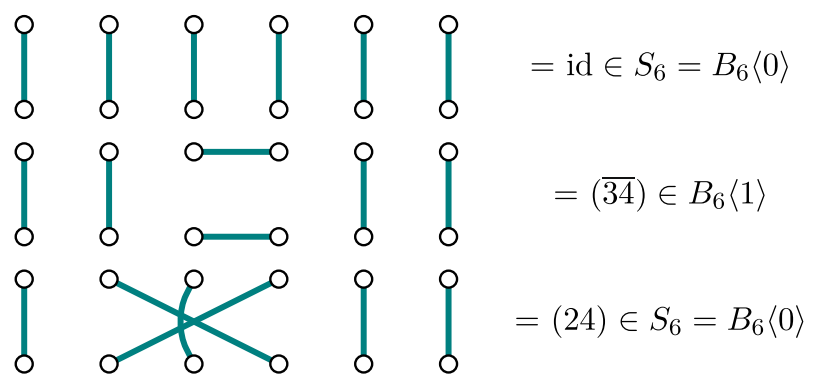

Fig. 4 The identity element, the element $(\overline{34}) \in B_{6}\langle 1\rangle$, and the transposition (24) $\in S_{6}=B_{6}\langle 0\rangle$
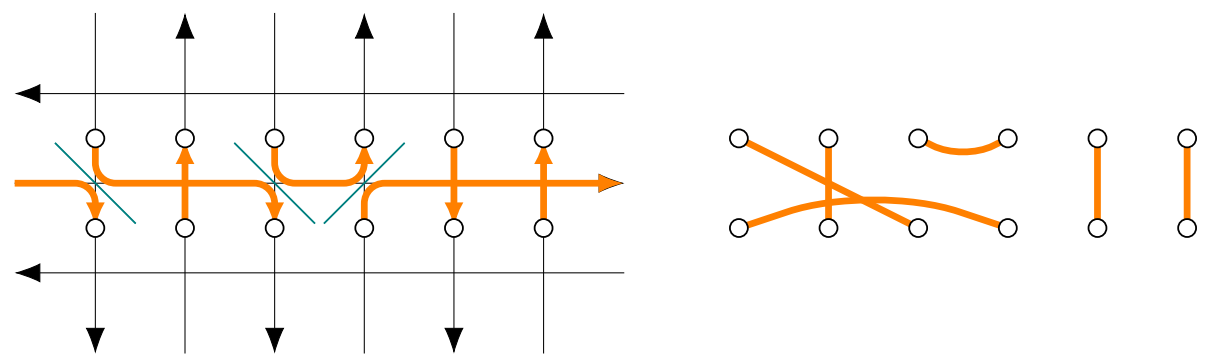

Fig. 5 An example of a configuration of mirrors $\sigma_{t}$ on street $s_{t}$ in the Manhattan model (left), and the resulting diagram $b\left(\sigma_{t}\right)$ (right)

group $S_{n}$, and the concatenation multiplication exactly reduces to the multiplication in $S_{n}$. So $\mathbb{C} S_{n}$ is a subalgebra of $\mathbb{B}_{n, 1}$.

We write id for the identity in $S_{n}$ - its diagram has all its edges vertical. We denote the transposition $S_{n}$ swapping $i$ and $j$ by $(i j)$, and we write $(\overline{i j})$ for the diagram with $i^{+}$connected to $j^{+}$, and $i^{-}$connected to $j^{-}$, and all other edges vertical. See Fig. 4 .

Finally, we remark that if $b$ has $k$ bars, and $c$ is any diagram in $B_{n}$, then $b c$ must have at least $k$ bars:

$$
b \in B_{n}\langle k\rangle \Rightarrow b c \in B_{n}^{k}
$$

Let us now see how the Brauer algebra can be used to describe the models. Let $n$ be even from hereon in. Observe that given a configuration $\sigma_{t}$ of mirrors on a street $s_{t}$ on the $n$ cylinder, the paths through the street form a diagram $b\left(\sigma_{t}\right) \in B_{n}$. See Fig. 5 for an illustration. Moreover, on any section of the cylinder, say, from street $s_{t_{1}}$ to $s_{t_{2}}$, given a configuration of mirrors $\sigma_{t_{1} \rightarrow t_{2}}$, the paths through those streets form a diagram $b\left(\sigma_{t_{1} \rightarrow t_{2}}\right)$. Crucially, we see that $b\left(\sigma_{t_{1} \rightarrow t_{2}}\right)=b\left(\sigma_{t_{1}}\right) \cdots b\left(\sigma_{t_{2}}\right)$, where the multiplication on the right hand side is in the Brauer algebra. See Fig. 6. 

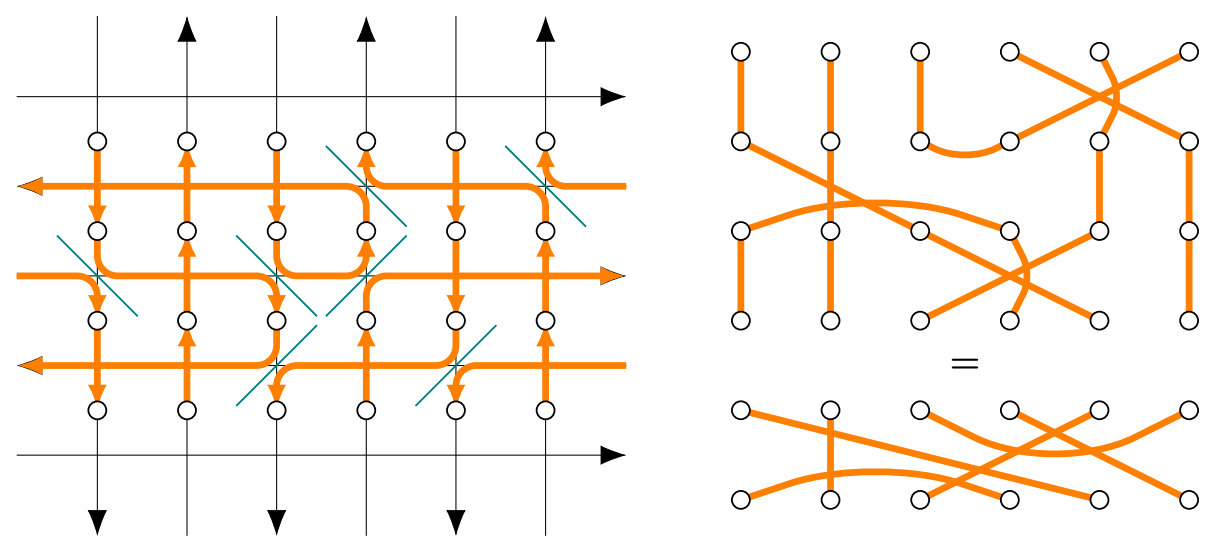

Fig. 6 The paths through three consecutive streets in the Manhattan model (left), the three resulting diagrams (upper right), and their product (lower right), which gives the paths through the union of the three streets

Let each $\sigma_{t}$, the configuration of mirrors on the $t^{\text {th }}$ street, be distributed according to the Manhattan or Mirror model. Then $b\left(\sigma_{t}\right)$ is a random diagram in $B_{n}$. We can think of the distribution of this random diagram as a (deterministic) element $Z_{(t)}$ of the algebra:

$$
Z_{(t)}=\sum_{g \in B_{n}} \mathbb{P}\left[b\left(\sigma_{t}\right)=g\right] \cdot g .
$$

The following lemma lets us describe the paths through any number of consecutive streets. Note that it does not use the specific distributions of the random diagrams given by different streets, it only uses that they are independent.

Lemma 2.1 The distribution of the random diagram $b\left(\sigma_{t_{1} \rightarrow t_{2}}\right)$ produced by the paths through streets $s_{t_{1}}, \ldots s_{t_{2}}$ is given by the Brauer algebra element:

$$
Z_{\left(t_{1}\right)} \cdots Z_{\left(t_{2}\right)}=\sum_{g \in B_{n}} \mathbb{P}\left[b\left(\sigma_{t_{1} \rightarrow t_{2}}\right)=g\right] \cdot g,
$$

where the multiplication on the left is in the Brauer algebra.

Proof We see that

$$
\begin{aligned}
\sum_{g \in B_{n}} \mathbb{P}\left[b\left(\sigma_{t_{1} \rightarrow t_{2}}\right)=g\right] \cdot g & =\sum_{g \in B_{n}} \mathbb{P}\left[b\left(\sigma_{t_{1}}\right) \cdots b\left(\sigma_{t_{2}}\right)=g\right] \cdot g \\
& =\sum_{g \in B_{n}} \sum_{g_{t_{1}} \cdots g_{t_{2}}=g} \mathbb{P}\left[b\left(\sigma_{t_{1}}\right)=g_{t_{1}}\right] \cdots \mathbb{P}\left[b\left(\sigma_{t_{2}}\right)=g_{t_{2}}\right] \cdot g_{t_{1}} \cdots g_{t_{2}} \\
& =Z_{\left(t_{1}\right)} \cdots Z_{\left(t_{2}\right)},
\end{aligned}
$$

where we use that the configurations on each street are independent.

We are interested in the highest (or most northerly) street reached by the paths starting at the first street $s_{1}$. One more than this is the first street which has no path connecting it to $s_{1}$. Using the notation above, this is the smallest $t$ such that the random diagram $b\left(\sigma_{1 \rightarrow t}\right)$ has no NS paths, i.e.:

$$
b\left(\sigma_{1 \rightarrow t}\right) \in B_{n}\left\langle\frac{n}{2}\right\rangle .
$$


Fig. 7 An example of paths through a street in the Manhattan model, with entrypoints coloured in yellow, and exitpoints in blue (Color figure online)

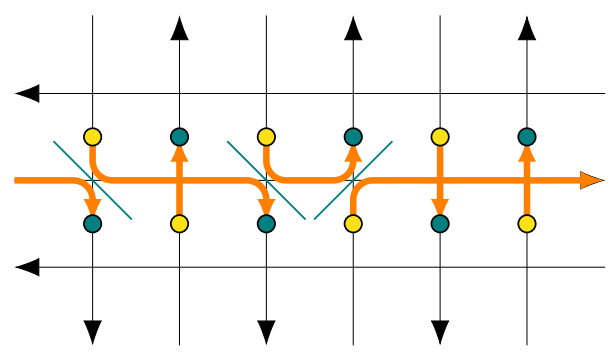

Let $*$ represent mat or mir. Let $\sigma_{*, t}$ (resp. $\sigma_{*, t_{1} \rightarrow t_{2}}$ ) denote the random configuration of mirrors on the street $s_{t}$ (resp. the streets $s_{t_{1}}, \ldots, s_{t_{2}}$ ), in the corresponding model. Now, in the Mirror model, the random configuration of mirrors $\sigma_{m i r, t}$ is iid for each street $s_{t}$. Let $Z_{m i r}$ be the distribution of the random diagram $b\left(\sigma_{m i r, t}\right)$ (as an element of the Brauer algebra) produced by the paths through this random configuration on one street. (Since the $\sigma_{m i r, t}$ are iid, $Z_{\text {mir }}$ is independent of $t$ ). We note that, from Lemma 2.1,

$$
Z_{m i r}^{t}=\sum_{g \in B_{n}} \mathbb{P}\left[b\left(\sigma_{m i r, 1 \rightarrow t}\right)=g\right] \cdot g .
$$

Let $V_{k}^{\text {mir }}$ be the random variable given by the smallest $t$ such that $b\left(\sigma_{m i r, 1 \rightarrow t}\right) \in B_{n}\langle k\rangle$ (this is the first street which has at most $n-2 k$ paths reaching it from the first street $\left.s_{1}\right)$. We are primarily interested in $V_{\frac{n}{2}}^{\text {mir }}$.

The Manhattan model is almost identical in this regard, with two differences. The first is that the random configuration of mirrors $\sigma_{m a t, t}$ on a street $s_{t}$ is dependent on whether the street is directed eastbound or westbound. We can let $Z_{(m a t, E)}, Z_{(m a t, W)}$ the corresponding elements of the Brauer algebra (similar to the mirror case, each only dependent on eastbound or westbound).

Secondly, the diagrams that arise in the Manhattan case actually live in a subalgebra of $\mathbb{B}_{n, 1}$. Note that each vertical column of the cylinder $\mathbb{Z} \times\{i\}, i=1, \ldots, n$, is southbound for $i$ odd, northbound for $i$ even. This means that on a chosen street, the vertices $i^{+}$for $i$ odd, and $i^{-}$for $i$ even, can be thought of as "entrypoints" to the street. Similarly, each $j^{+}$for $j$ even, $j^{-}$for $j$ odd can be thought of as "exitpoints" to the street. In particular, in the diagram which results from the street, exitpoints must be connected to entrypoints. This condition can also be thought of as: a NS path must connect vertices of the same parity, and a bar must connect vertices of different parity. See Fig. 7.

Let $M_{n}$ be the set of diagrams which satisfy the requirement that exitpoints are only connected to entrypoints, and let $\mathbb{M}_{n, 1}$ be the (formal) complex span of $M_{n}$. This space $\mathbb{M}_{n, 1}$ is a subalgebra of $\mathbb{B}_{n, 1}$, indeed it is a special case of the Walled Brauer algebra. See [5]. Similar to the full algebra, let $M_{n}\langle k\rangle$ be the set of diagrams in $M_{n}$ with $k$ bars, and let $M_{n}^{k}$ be those with at least $k$ bars.

Let us assume that the first street, $s_{1}$, is eastbound. Now let $V_{k}^{\text {mat }}$ be the random variable given by the smallest $t$ such that $b\left(\sigma_{\text {mat }, 1 \rightarrow t}\right) \in M_{n}\langle k\rangle$. Note that the distribution of the random diagram $b\left(\sigma_{\text {mat }, 1 \rightarrow t}\right)$ is described by the element of $\mathbb{M}_{n, 1}$ : 


$$
\begin{aligned}
& \sum_{g \in B_{n}} \mathbb{P}\left[b(\sigma_{\text {mat }, 1 \rightarrow t)=g] \cdot g}=\underbrace{Z_{(\text {mat }, E)} Z_{(\text {mat }, W)} Z_{(\text {mat }, E)} \cdots}_{\mathrm{t} \text { terms }}\right. \\
&= \begin{cases}\left(Z_{(\text {mat }, E)} Z_{(\text {mat }, W)}\right)^{\frac{t}{2}} & t \text { even } \\
\left(Z_{(\text {mat }, E)} Z_{(\text {mat }, W)}\right)^{\frac{t-1}{2}} Z_{(\text {mat }, E)} & t \text { odd },\end{cases}
\end{aligned}
$$

where the equality is included for clarity. We are primarily interested in $V_{\frac{n}{2}}^{m a t}$.

Now recall that our method is to condition on there being at most two mirrors per street. Let $U_{\leq 2}^{(t)}$ be the event that there are at most two mirrors on a street $s_{t}$ (this event has probability $\mathbb{P}\left[U_{\leq 2}\right]$ independent of the street, and the model we are considering). Let $\sigma_{*, t}^{\leq 2}$ (resp. $\sigma_{*, t_{1} \rightarrow t_{2}}$ ) be the random configuration of mirrors on the street $t$ (resp. the streets $s_{t_{1}}, \ldots, s_{t_{2}}$ ) when conditioning on $U_{\leq 2}$. Let $X_{\text {mir }}, X_{m a t}$ be the elements $Z_{m i r}, Z_{(m a t, E / W)}$, produced when conditioning on $U_{\leq 2}$, respectively. (In the Manhattan case, it actually does not matter whether the street is eastbound or westbound). That is, $X_{\text {mir }}$ and $X_{\text {mat }}$ describe the distributions of $b\left(\sigma_{\text {mir }, t}^{\leq 2}\right)$ and $b\left(\sigma_{\text {mat }, t}^{\leq 2}\right)$, respectively; for $*$ denoting mir or mat,

$$
X_{*}^{t}=\sum_{g \in B_{n}} \mathbb{P}\left[b\left(\sigma_{*, 1 \rightarrow t}^{\leq 2}\right)=g\right] \cdot g .
$$

We can write these elements explicitly:

$$
X_{\text {mir }}=\frac{(1-p)^{n-2}}{\mathbb{P}\left[U_{\leq 2}\right]}\left[\left(n p(1-p)+(1-p)^{2}\right) \cdot \mathrm{id}+\frac{p^{2}}{2}\left(\sum_{1 \leq i<j \leq n}(i j)+(\overline{i j})\right)\right],
$$

and very similarly:

$$
X_{\text {mat }}=\frac{(1-p)^{n-2}}{\mathbb{P}\left[U_{\leq 2}\right]}\left[\left(n p(1-p)+(1-p)^{2}\right) \cdot \mathrm{id}+p^{2}\left(\sum_{j-i \text { even }}(i j)+\sum_{j-i \text { odd }}(\overline{i j})\right)\right]
$$

where we recall that the diagrams $(i j),(\overline{i j})$, and id are given in Fig. 4. Note that $Z_{\text {mir }}$, $Z_{(m a t, E / W)}$ can also be explicitly written down as elements of the Brauer algebra (for any $p)$, they are just far more unwieldy.

Similar to above, let $*$ denote mir or $m a t$, and define $W_{k}^{*}$ to be the random variable given by the smallest $t$ such that $b\left(\sigma_{1 \rightarrow t}^{\leq 2}\right) \in B_{n}\left\langle\frac{n}{2}\right\rangle$. In the next section, we give bounds on how large $W_{\frac{n}{2}}^{*}$ can be, and then we transfer these bounds to $V_{\frac{n}{2}}^{*}$.

\section{Results}

Let us first prove part (b) of Theorem 1.1. Let $*$ denote mir or mat. Let $G$ be a geometric random variable with parameter $p^{2}$. We first show that $\mathbb{P}\left[V_{\frac{n}{2}}^{*} \leq x\right] \leq \mathbb{P}[G \leq x]$, for all $x \geq 0$.

Assume that $b\left(\sigma_{*, 1 \rightarrow t}\right) \notin B_{n}\left\langle\frac{n}{2}\right\rangle$, that is, after $t$ streets, there are at least two remaining NS paths. Consider the probability $\mathbb{P}\left[b\left(\sigma_{*, 1 \rightarrow t+1}\right) \in B_{n}\left\langle\frac{n}{2}\right\rangle\right]$, that after the next street, no NS paths remain. In order for $b\left(\sigma_{*, 1 \rightarrow t+1}\right) \in B_{n}\left\langle\frac{n}{2}\right\rangle$ to hold, there certainly must be a mirror 
on $s_{t+1}$ reflecting each of the remaining NS paths - since there are at least two of these, the probability of this is at most $p^{2}$. Hence we can say that, given that $b\left(\sigma_{*, 1 \rightarrow t}\right) \notin B_{n}\left\langle\frac{n}{2}\right\rangle$,

$$
\mathbb{P}\left[b\left(\sigma_{*, 1 \rightarrow t+1}\right) \in B_{n}\left\langle\frac{n}{2}\right\rangle\right] \leq p^{2} .
$$

Now we can easily couple the process with one which enters $B_{n}\left\langle\frac{n}{2}\right\rangle$ at each step with probability exactly $p^{2}$. The time taken for this process to enter $B_{n}\left\langle\frac{n}{2}\right\rangle$ can be described by $G$, and our claim $\mathbb{P}\left[V_{\frac{n}{2}}^{*} \leq x\right] \leq \mathbb{P}[G \leq x]$ follows. Now for $p \leq \frac{1}{2}$,

$$
\mathbb{P}\left[V_{\frac{n}{2}}^{*} \leq \alpha p^{-2}\right] \leq \mathbb{P}\left[G \leq \alpha p^{-2}\right]=1-\left(1-p^{2}\right)^{\alpha p^{-2}} \leq 2 \alpha,
$$

the last inequality following from both functions taking the value 0 at $\alpha=0$, and the differential of the first function being $-\left(1-p^{2}\right)^{\alpha p^{-2}} \log \left(\left(1-p^{2}\right)^{p^{-2}}\right)$, whose value is less than 2 at $\alpha=0$ and decreasing as $\alpha$ increases. This completes the proof of part (b).

The rest of this section proves part (a) of Theorem 1.1. We return to our simplified model, assuming at most two mirrors on each street. Observe that if the random diagram $b\left(\sigma_{*, t}^{\leq 2}\right)$ is multiplied with a diagram $g$ which has $k$ bars, the probability that the result has $k+1$ bars is independent of the chosen diagram $b$. This is made precise in the following lemma.

Lemma 3.1 (a) Let $g \in B_{n}\langle k\rangle$, a diagram with $k$ bars. Then $g \cdot b\left(\sigma_{*, t}^{\leq 2}\right) \in B_{n}\langle k\rangle \cup B_{n}\langle k+1\rangle$, and

$$
g_{n, p, k}^{\text {mir }}:=\mathbb{P}\left[g \cdot b\left(\sigma_{\text {mir }, t}^{\leq 2}\right) \in B_{n}\langle k+1\rangle\right]=\frac{1}{\mathbb{P}\left[U_{\leq 2}\right]} \frac{p^{2}}{2}(1-p)^{n-2}\left(\begin{array}{c}
n-2 k \\
2
\end{array}\right) .
$$

(b) Let $g \in M_{n}\langle k\rangle$, a diagram with $k$ bars. Then $g \cdot b\left(\sigma_{m a t, t}^{\leq 2}\right) \in M_{n}\langle k\rangle \cup M_{n}\langle k+1\rangle$, and

$$
g_{n, p, k}^{\text {mat }}:=\mathbb{P}\left[g \cdot b\left(\sigma_{m a t, t}^{\leq 2}\right) \in M_{n}\langle k+1\rangle\right]=\frac{1}{\mathbb{P}\left[U_{\leq 2}\right]} p^{2}(1-p)^{n-2}\left(\frac{n}{2}-k\right)^{2} .
$$

Proof Let us do part (a) first. Let $g \in B_{n}\langle k\rangle$. It is clear that $g(i j) \in B_{n}\langle k\rangle$. Further, $g(\overline{i j}) \in$ $B_{n}\langle k+1\rangle$ iff the vertices $i^{-}$and $j^{-}$in $g$ lie on NS paths. There are $\left(\begin{array}{c}n-2 k \\ 2\end{array}\right)$ such pairs. So,

$$
\mathbb{P}\left[b\left(\sigma_{\text {mir }, t}^{\leq 2}\right)=(\overline{i j}), i^{-}, j^{-} \text {on NS paths in } g\right]=\frac{p^{2}}{2} \frac{(1-p)^{n-2}}{\mathbb{P}\left[U_{\leq 2}\right]}\left(\begin{array}{c}
n-2 k \\
2
\end{array}\right)=g_{n, p, k}^{m i r} .
$$

Part (b) follows very similarly. Let $g \in M_{n}\langle k\rangle$. Then $g(\overline{i j}) \in M_{n}\langle k+1\rangle$ iff the vertices $i^{-}$and $j^{-}$in $g$ lie on NS paths. There are $\left(\frac{n}{2}-k\right)^{2}$ such pairs. So,

$$
\mathbb{P}\left[b\left(\sigma_{\text {mat }, t}^{\leq 2}\right)=(\overline{i j}), i^{-}, j^{-} \text {on NS paths in } g\right]=\frac{p^{2}(1-p)^{n-2}}{\mathbb{P}\left[U_{\leq 2}\right]}\left(\frac{n}{2}-k\right)^{2}=g_{n, p, k}^{\text {mat }} .
$$

Let $*$ denote mir or mat. Let $w_{k}^{*}=W_{k+1}^{*}-W_{k}^{*}$; this is the number of streets we have to wait between the $k^{\text {th }}$ and the $k+1^{\text {th }}$ bar being added to the random diagram. Lemma 3.1 shows that $w_{k}^{*}$ is a geometric random variable, with parameter $g_{n, p, k}^{*}$. Note that $W_{\frac{n}{2}}^{*}=\sum_{k=0}^{\frac{n}{2}-1} w_{k}^{*}$. The next theorem bounds the probability that $W_{\frac{n}{2}}^{*}$ is large.

Theorem 3.2 Let $*$ represent mat or mir. Let $p \leq \mathrm{Cn}^{-1}, C$ a constant. Then for all $\alpha>0$,

$$
\mathbb{P}\left[W_{\frac{n}{2}}^{*} \geq \alpha p^{-2}\right] \leq A_{*} e^{-\frac{1}{4 C_{2}} \alpha},
$$


where $A_{m i r}=\cosh (\pi)$, and $A_{m a t}=\frac{\sinh (\pi)}{\pi}$, and $C_{2}=\frac{1}{2} C^{2}+C+1$.

Proof of Theorem 3.2 Let us look at the Manhattan case. We first note that, using $p<C n^{-1}$,

$$
\begin{aligned}
g_{n, p, k}^{\text {mat }} & =\frac{\left(\frac{n}{2}-k\right)^{2}}{\frac{1}{2} n^{2}-\frac{1}{2} n+n p^{-1}-n+p^{-2}-2 p^{-1}+1} \\
& \geq \frac{\left(\frac{n}{2}-k\right)^{2}}{\left(\frac{1}{2} C^{2}+C+1\right) p^{-2}} \\
& =\left(\frac{n}{2}-k\right)^{2} C_{2}^{-1} p^{2} .
\end{aligned}
$$

Now, recall that $W_{\frac{n}{2}}^{\text {mat }}=\sum_{k=0}^{\frac{n}{2}-1} w_{k}^{\text {mat }}$, and that $w_{k}^{\text {mat }}$ are independent and geometrically distributed with parameter $g_{n, p, k}^{m a t}$. Recall also that the moment generating function of a geometric random variable $G$ with parameter $\lambda$ is given by

$$
\mathbb{E}\left[e^{t G}\right]=\frac{\lambda}{1-(1-\lambda) e^{t}}
$$

for $t<-\log (1-\lambda)$. This inequality holds when we set $t=\frac{p^{2}}{4 C_{2}}$ and $\lambda=p^{2}$, since $C_{2}=\frac{1}{2} C^{2}+C+1 \geq \frac{1}{2}$. We have, using Chebyshev's exponential inequality,

$$
\begin{aligned}
\mathbb{P}\left[W_{\frac{n}{2}}^{\text {mat }} \geq \alpha p^{-2}\right] & \leq e^{-\frac{1}{4 C_{2}} \alpha} \mathbb{E}\left[e^{\frac{p^{2}}{4 C_{2}} W_{\frac{n}{2}}^{\text {mat }}}\right] \\
& =e^{-\frac{1}{4 C_{2}} \alpha} \prod_{k=0}^{\frac{n}{2}-1}\left(\frac{g_{n, p, k}^{\text {mat }}}{1-\left(1-g_{n, p, k}^{\text {mat }}\right) e^{\frac{p^{2}}{4 C_{2}}}}\right) \\
& =e^{-\frac{1}{4 C_{2}} \alpha} \prod_{k=0}^{\frac{n}{2}-1}\left(1+\frac{e^{\frac{p^{2}}{4 C_{2}}}-1}{1-\left(1-g_{n, p, k}^{m a t}\right) e^{\frac{p^{2}}{4 C_{2}}}}\right) .
\end{aligned}
$$

Using $e^{t} \leq 2 t+1$, (which holds for $t=\frac{p^{2}}{4 C_{2}}<1$, which in turn always holds, since $C_{2} \geq \frac{1}{2}$ ), we have

$$
\begin{aligned}
1-\left(1-g_{n, p, k}^{\text {mat }}\right) e^{\frac{p^{2}}{4 C_{2}}} & \geq 1-\left(1-\left(\frac{n}{2}-k\right)^{2} C_{2}^{-1} p^{2}\right)\left(\frac{1}{2} C_{2}^{-1} p^{2}+1\right) \\
& \geq C_{2}^{-1} p^{2}\left(\left(\frac{n}{2}-k\right)^{2}-\frac{1}{2}\right)
\end{aligned}
$$


which gives:

$$
\begin{aligned}
\mathbb{P}\left[W_{\frac{n}{2}}^{\text {mat }} \geq \alpha p^{-2}\right] & \leq e^{-\frac{1}{4 C_{2}} \alpha} \prod_{k=0}^{\frac{n}{2}-1}\left(1+\frac{\frac{1}{2} C_{2}^{-1} p^{2}}{C_{2}^{-1} p^{2}\left(\left(\frac{n}{2}-k\right)^{2}-\frac{1}{2}\right)}\right) \\
& =e^{-\frac{1}{4 C_{2}} \alpha} \prod_{k=1}^{\frac{n}{2}}\left(1+\frac{1}{2 k^{2}-1}\right) \\
& \leq e^{-\frac{1}{4 C_{2}} \alpha} \prod_{k=1}^{\frac{n}{2}}\left(1+\frac{1}{k^{2}}\right) \\
& \leq \frac{\sinh \pi}{\pi} e^{-\frac{1}{4 C_{2}} \alpha},
\end{aligned}
$$

as desired. In the last inequality we used the product formula $\sin (\pi z)=\pi z \prod_{\nu=1}^{\infty}\left(1-\frac{z^{2}}{v^{2}}\right)$, with $z=i$.

The Mirror model case is almost identical; all the above working is the same except the expression $\left(\frac{n}{2}-k\right)^{2}$ is replaced with $\frac{1}{2}\left(\begin{array}{c}n-2 k \\ 2\end{array}\right)$. This gives

$$
\begin{aligned}
\mathbb{P}\left[W_{\frac{n}{2}}^{\text {mat }} \geq \alpha p^{-2}\right] & \leq e^{-\frac{1}{4 C_{2}} \alpha} \prod_{k=0}^{\frac{n}{2}-1}\left(1+\frac{\frac{1}{2}}{\frac{1}{2}\left(\begin{array}{c}
n-2 k \\
2
\end{array}\right)-\frac{1}{2}}\right) \\
& \leq e^{-\frac{1}{4 C_{2}} \alpha} \prod_{k=1}^{\frac{n}{2}}\left(1+\frac{4}{2 k(2 k-1)}\right) \\
& \leq \cosh (\pi) e^{-\frac{1}{4 C_{2}} \alpha}
\end{aligned}
$$

as desired, where for the last equality we used the product formula $\cos (\pi z)=\prod_{\nu=1}^{\infty}(1-$ $\left.\frac{4 z^{2}}{(2 v-1)^{2}}\right)$, with $z=i$.

We can now compare the full models with the models assuming at most two mirrors per street. Let $t \in \mathbb{N}$. Let $\tau(t)$ be the random variable given by the number of the first $t$ streets which have at most 2 mirrors. We see that $\tau(t)$ is binomially distributed with parameters $\left(t, \mathbb{P}\left[U_{\leq 2}\right]\right)$. Essentially what we would like to say is that if we omit each street which has more than 2 mirrors, we do not, in distribution, add any bars.

This sounds like it should follow from the remark (1), but it is more subtle. Let us illustrate why: certainly if the product of two diagrams $a b$ has $k$ bars, then we can conclude that each of $a$ and $b$ have no more than $k$ bars. However, if $a b c$ has $k$ bars, it is very possible that $a c$ has more than $k$ bars. So, when removing factors from the middle of a product, there is more to be proved.

Lemma 3.3 Let $*$ denote mir or mat. Then $\mathbb{P}\left[V_{k}^{*} \leq t\right] \geq \mathbb{P}\left[W_{k}^{*} \leq \tau(t)\right]$.

Recall that $g \in B_{n}^{k}$ iff $g$ has at least $k$ bars, and $g \in M_{n}^{k}$ similar. Note that $V_{k}^{*} \leq t$ iff $b\left(\sigma_{*, 1 \rightarrow t}\right) \in B_{n}^{k}$; similar for $W_{k}^{*}$. So Lemma 3.3 can be rewritten as:

$$
\mathbb{P}\left[b\left(\sigma_{*, 1 \rightarrow t}\right) \in B_{n}^{k}\right] \geq \mathbb{P}\left[b\left(\sigma_{*, 1 \rightarrow \tau(t)}^{\leq 2}\right) \in B_{n}^{k}\right] .
$$

We postpone the proof of Lemma 3.3, and first see how it is implemented, combining with Theorem 3.2 in proving part (a) of Theorem 1.1. 
Proof of part (a) of Theorem 1.1 Recall that we assume $p \leq C n^{-1}, C$ a constant. We approximate $b\left(\sigma_{*, 1 \rightarrow t}\right)$ with $b\left(\sigma_{*, 1 \rightarrow \tau(t)}^{\leq 2}\right)$, that is, we approximate by ignoring streets which have more than two mirrors. Since the expected number of mirrors per street is at most $C$, we expect (at least for $C$ small) the proportion of streets with at most two mirrors to be large. Indeed:

$$
\begin{aligned}
\lim _{n \rightarrow \infty} \mathbb{P}\left[U_{\leq 2}\right] & =\lim _{n \rightarrow \infty}(1-p)^{n-2}\left((1-p)^{2}+n p(1-p)+\left(\begin{array}{l}
n \\
2
\end{array}\right) p^{2}\right) \\
& \geq \lim _{n \rightarrow \infty}(1-p)^{c p^{-1}-2}\left((1-p)(1-p+C)+\frac{C}{2}(C-p)\right),
\end{aligned}
$$

the limit of which is $\left(\frac{1}{e}\right)^{C}\left(1+C+\frac{C^{2}}{2}\right)=: C_{3}$. We can pick $n \in \mathbb{N}$ such that $\mathbb{P}\left[U_{\leq 2}\right]>$ $\frac{3 C_{3}}{4}$. Recalling $\tau(t)$ is binomially distributed with parameters $\left(t, \mathbb{P}\left[U_{\leq 2}\right]\right)$, by Hoeffding's inequality,

$$
\mathbb{P}\left[\tau(t) \leq \frac{C_{3} t}{2}\right] \leq \exp \left[-2 t\left(\mathbb{P}\left[U_{\leq 2}\right]-\frac{C_{3}}{2}\right)^{2}\right] \leq \exp \left[-2 t\left(\frac{C_{3}}{4}\right)^{2}\right],
$$

for $n$ large enough.

Let $t=\alpha p^{-2}$. Now using Lemma 3.3,

$$
\begin{aligned}
\mathbb{P}\left[V_{k}^{*} \geq \alpha p^{-2}\right] & \leq \mathbb{P}\left[W_{k}^{*} \geq \tau\left(\alpha p^{-2}\right)\right] \\
& \leq \mathbb{P}\left[W_{k}^{*} \geq \frac{C_{3}}{2} \alpha p^{-2}\right]+\mathbb{P}\left[\tau(t) \leq \frac{1}{2} \alpha p^{-2}\right] \\
& \leq A_{*} \exp \left[-\frac{C_{3}}{8 C_{2}} \alpha\right]+\exp \left[-2 \alpha p^{-2}\left(\frac{C_{3}}{4}\right)^{2}\right] \\
& \leq 2 A_{*} \exp \left[-\frac{1}{8 e^{C}} \alpha\right]
\end{aligned}
$$

where the second to last inequality is from Theorem 3.2 and Eq. (3), and the last is for $p$ small enough.

It remains to prove Lemma 3.3.

Proof of Lemma 3.3 We prove the inequality (2). We fix $n$, and work by induction on $t$ and $k$. The inequality is trivially true for $k=0$ (and any $t$ ), and for $t=1$ (and any $k$ ).

Assume the Lemma holds for the parameters $(t-1, k),(t-1, k-1)$, and $(t, k-1)$. The left hand side of Eq. (2) is:

$$
\begin{aligned}
\mathbb{P}\left[b\left(\sigma_{*, 1 \rightarrow t}\right) \in B_{n}^{k}\right]= & \mathbb{P}\left[b\left(\sigma_{*, 1 \rightarrow t-1}\right) b\left(\sigma_{*, t}\right) \in B_{n}^{k} \mid U_{>2}^{(t)}\right] \cdot\left(1-\mathbb{P}\left[U_{\leq 2}\right]\right) \\
& +\mathbb{P}\left[b\left(\sigma_{*, 1 \rightarrow t-1}\right) b\left(\sigma_{*, t}\right) \in B_{n}^{k} \mid U_{\leq 2}^{(t)}\right] \cdot \mathbb{P}\left[U_{\leq 2}\right] \\
\geq & \mathbb{P}\left[b\left(\sigma_{*, 1 \rightarrow t-1}\right) \in B_{n}^{k} \mid U_{>2}^{(t)}\right] \cdot\left(1-\mathbb{P}\left[U_{\leq 2}\right]\right) \\
& +\mathbb{P}\left[b\left(\sigma_{*, 1 \rightarrow t-1}\right) b\left(\sigma_{*, t}^{\leq 2}\right) \in B_{n}^{k} \mid U_{\leq 2}^{(t)}\right] \cdot \mathbb{P}\left[U_{\leq 2}\right],
\end{aligned}
$$

where we have noted that the number of bars in the product $b\left(\sigma_{*, 1 \rightarrow t-1}\right) b\left(\sigma_{*, t}\right)$ cannot be less that in $b\left(\sigma_{*, 1 \rightarrow t-1}\right)$, and that $b\left(\sigma_{*, t}\right)$ is equal to $b\left(\sigma_{*, t}^{\leq 2}\right)$ when conditioned on $U_{\leq 2}^{(t)}$. Now 
the above is at least:

$$
\begin{aligned}
& \geq \mathbb{P}\left[b\left(\sigma_{*, 1 \rightarrow \tau(t-1)}^{\leq 2}\right) \in B_{n}^{k} \mid U_{>2}^{(t)}\right] \cdot\left(1-\mathbb{P}\left[U_{\leq 2}\right]\right)+\mathbb{P}\left[b\left(\sigma_{*, 1 \rightarrow \tau(t)}^{\leq 2}\right) \in B_{n}^{k} \mid U_{\leq 2}^{(t)}\right] \cdot \mathbb{P}\left[U_{\leq 2}\right] \\
& =\mathbb{P}\left[b\left(\sigma_{*, 1 \rightarrow \tau(t)}^{\leq 2}\right) \in B_{n}^{k}\right]
\end{aligned}
$$

where in the inequality we used the inductive assumption on $t$ and the final Lemma below, and in the equality we used the fact that under $U_{>2}^{(t)}, \tau(t)=\tau(t-1)$. The proof of the final Lemma therefore concludes the whole proof.

Lemma 3.4 We have that $\mathbb{P}\left[b\left(\sigma_{*, 1 \rightarrow t-1}\right) b\left(\sigma_{*, t}^{\leq 2}\right) \in B_{n}^{k} \mid U_{\leq 2}^{(t)}\right] \geq \mathbb{P}\left[b\left(\sigma_{*, 1 \rightarrow \tau(t)}^{\leq 2}\right) \in B_{n}^{k} \mid U_{\leq 2}^{(t)}\right]$.

Proof To prove the claim, we split the left hand term based on whether or not $b\left(\sigma_{*, t}^{\leq 2}\right)$ adds a bar to $b\left(\sigma_{*, 1 \rightarrow t-1}\right)$ :

$$
\begin{aligned}
\text { LHS } & =\mathbb{P}\left[b\left(\sigma_{*, 1 \rightarrow t-1}\right) \in B_{n}^{k-1} \mid U_{\leq 2}^{(t)}\right] \cdot g_{n, p, k}^{*}+\mathbb{P}\left[b\left(\sigma_{*, 1 \rightarrow t-1}\right) \in B_{n}^{k} \mid U_{\leq 2}^{(t)}\right] \\
& \geq \mathbb{P}\left[b\left(\sigma_{*, 1 \rightarrow \tau(t-1)}^{\leq 2}\right) \in B_{n}^{k-1} \mid U_{\leq 2}^{(t)}\right] \cdot g_{n, p, k}^{*}+\mathbb{P}\left[b\left(\sigma_{*, 1 \rightarrow \tau(t-1)}^{\leq 2}\right) \in B_{n}^{k} \mid U_{\leq 2}^{(t)}\right] \\
& =\mathbb{P}\left[b\left(\sigma_{*, 1 \rightarrow \tau(t-1)}^{\leq 2}\right) b\left(\sigma_{*, t}^{\leq 2}\right) \in B_{n}^{k} \mid U_{\leq 2}^{(t)}\right],
\end{aligned}
$$

where in the inequality we used the inductive assumption on $t$ and $k$. Now recalling that under $U_{\leq 2}, \tau(t)=\tau(t-1)+1$, the result follows. This concludes the proof of Lemma 3.3 and part (a) of Theorem 1.1.

Acknowledgements I would like to thank Sasha Sodin for many useful discussions.

Open Access This article is licensed under a Creative Commons Attribution 4.0 International License, which permits use, sharing, adaptation, distribution and reproduction in any medium or format, as long as you give appropriate credit to the original author(s) and the source, provide a link to the Creative Commons licence, and indicate if changes were made. The images or other third party material in this article are included in the article's Creative Commons licence, unless indicated otherwise in a credit line to the material. If material is not included in the article's Creative Commons licence and your intended use is not permitted by statutory regulation or exceeds the permitted use, you will need to obtain permission directly from the copyright holder. To view a copy of this licence, visit http://creativecommons.org/licenses/by/4.0/.

\section{References}

1. Beamond, E.J., Cardy, J., Chalker, J.T.: Quantum and classical localisation, the spin quantum Hall effect and generalisations. Phys. Rev. B 65(21), 214301 (2002)

2. Beamond, E.J., Owczarek, A.L., Cardy, J.: Quantum and classical localization and the Manhattan lattice. J. Phys. A 36(41), 10251-10267 (2003)

3. Brauer, Richard: On algebras which are connected with the semisimple continuous groups. Ann. Math. 38(4), 857-872 (1937)

4. Brown, William P.: The semisimplicity of $\omega_{n}^{f}$. Ann. Math. 63(2), 324-335 (1956)

5. Cox, A., De Visscher, M., Doty, S., Martin, P.: On the blocks of the walled Brauer algebra. J. Algebra 320(1), 169-212 (2008)

6. Cox, A., De Visscher, M., Martin, P.: The blocks of the Brauer algebra in characteristic zero. Represent. Theory 13, 272-308 (2009)

7. Di Francesco, P., Zinn-Justin, P.: Around the Razumov-Stroganov conjecture: proof of a multi-parameter sum rule. Electron. J. Combin. 12, R6 (2005)

8. Di Francesco, P., Zinn-Justin, P.: Inhomogeneous model of crossing loops and multidegrees of some algebraic varieties. Commun. Math. Phys. 262(2), 459-487 (2006)

9. Grimmett, G.: Percolation. Springer, New York (1989) 
10. Kozma, G., Sidoravicius, V.: Lower bound for the escape probability in the Lorentz mirror model on $\mathbb{Z}^{2}$. Isr. J. Math. 209(2), 683-685 (2015)

11. Li, L.: On the Manhattan pinball problem (2020). arXiv:2006.10797

12. Li, L.: Polynomial bound for the localization length of Lorentz mirror model on the $1 \mathrm{~d}$ cylinder (2020). arXiv:2010.05900

13. Martins, M.J., Nienhuis, B., Rietman, R.: Intersecting loop model as a solvable super spin chain. Phys. Rev. Lett. 81(3), 504-507 (1998)

14. Nienhuis, B., Rietman, R.: A solvable model for intersecting loops (1993). arXiv:hep-th/9301012

15. Razumov, A.V., Stroganov, Yu.G.: Combinatorial nature of the ground-state vector of the $\mathrm{O}(1)$ loop model. Teoret. Mat. Fiz. 138(3), 395-400 (2004)

16. Ruijgrok, Th.W., Cohen, E.G.D.: Deterministic lattice gas models. Phys. Lett. A 133(7-8), 415-418 (1988)

17. Spencer, T.: Duality, statistical mechanics, and random matrices. In: Current Developments in Mathematics 2012, pp. 229-260. International Press, Somerville(2013)

18. Wenzl, H.: On the structure of Brauer's centralizer algebras. Ann. Math. (2) 128(1), 173-193 (1988)

19. Ziff, R.M., Kong, X.P., Cohen, E.G.D.: Lorentz lattice-gas and kinetic-walk model. Phys. Rev. A 44, 2410-2428 (1991)

Publisher's Note Springer Nature remains neutral with regard to jurisdictional claims in published maps and institutional affiliations. 